This is an electronic reprint of the original article. This reprint may differ from the original in pagination and typographic detail.

Author(s): Terziyan, Vagan; Golovianko, Mariia; Shevchenko, Oleksandr

Title: $\quad$ Semantic Portal as a Tool for Structural Reform of the Ukrainian Educational System

Year: $\quad 2015$

Version:

Please cite the original version:

Terziyan, V., Golovianko, M., \& Shevchenko, O. (2015). Semantic Portal as a Tool for Structural Reform of the Ukrainian Educational System. Information Technology for Development, 21(3), 381-402. https://doi.org/10.1080/02681102.2014.899955

All material supplied via JYX is protected by copyright and other intellectual property rights, and duplication or sale of all or part of any of the repository collections is not permitted, except that material may be duplicated by you for your research use or educational purposes in electronic or print form. You must obtain permission for any other use. Electronic or print copies may not be offered, whether for sale or otherwise to anyone who is not an authorised user. 


\title{
Semantic Portal as a Tool for Structural Reform of Ukrainian Educational System ${ }^{1}$
}

\author{
Vagan Terziyan ${ }^{1}$, Mariia Golovianko ${ }^{2}$, Oleksandr Shevchenko ${ }^{2}$ \\ ${ }^{1}$ Department of Mathematical Information Technology, University of Jyväskylä, Finland, e- \\ mail:vagan.terziyan@jyu.fi
}

${ }^{2}$ Department of Artificial Intelligence, Kharkiv National University of Radioelectronics, Ukraine

Vagan Terziyan, Professor in Distributed Systems, Department of Mathematical Information Technology, University of Jyvaskyla (Finland). He got his M.Sc. Degree in Applied Mathematics (1981), PhD in Artificial Intelligence (1985), and Habilitation (1993) in Knowledge Engineering, - all from Kharkov National University of Radioelectronics (KNURE). He has also academic title of Professor in Software Engineering granted by the State Attestation Committee of Ukraine. His area of expertise includes distributed self-managed systems, semantic Web, agent-based systems, knowledge management and engineering, education management and others.

Mariia Golovianko graduated in 2006 from KNURE as M.Sc. in Artificial Intelligence. In 2011 she defended a PhD thesis: Ontology-Based Methods and Models of Web-Content Verification. Now she works as a Senior Lecturer at Artificial Intelligence Department of KNURE and also as an expert in development and implementation of semantic technologies, AI and Web 2.0 into educational and scientific processes. She took part in several national and international projects on ontology-based resource management and quality assurance in education.

Oleksandr Shevchenko graduated in 2002 from KNURE as M.Eng. in Intelligent Decision-Support Systems. In 2006 defended a PhD thesis: Intelligent System for Ontology-Based Management and Integration of Heterogeneous Information. Now works as an Assoc. Prof. at Artificial Intelligence Department of KNURE and also as an expert in self-managed systems, educational ontologies and portals, information systems and software engineering. He took part in several national and international projects as an expert in ontology engineering, cloud computing and as educational portals architect.

1 Terziyan V., Golovianko M., Shevchenko O., Semantic Portal as a Tool for Structural Reform of Ukrainian Educational System, In: P. Soja and P. Rupino da Cunha (eds.), ICT in Transition Economies: Narrowing the Research Gap to Developed Countries, Special Issue in: Information Technology for Development, Taylor \& Francis Group, 2014 (this draft is currently under review). 


\title{
Semantic Portal as a Tool for Structural Reform of Ukrainian Educational System
}

\begin{abstract}
Education is recognized as a fundamental enabler of human development. The adoption of ICTs by education (especially in developing countries) contributes to educational system reforms, in addition to the traditional advantages, such as social openness and accessibility. Yet the academic community has not studied sufficiently the challenging context in which ICTs are used as instruments for the reform of inefficient, and sometimes even corrupted, educational systems rather than just as means for smarter classrooms, remote access, or content management. The object for this study is Ukrainian higher education and its quality assurance system, which appears to be neither flexible nor transparent. We offer an ICT solution to provide the needed transparency. We show that such transparency is urgently needed for the structural reform of this system, in order to empower people and recover their trust and respect towards higher education. The solution is based on ontology-driven portal as a digital ecosystem for national quality assurance. We show how the content of the portal can be managed and verified based on users' social activity and reputation management, and how the quality evaluations of different players can be made with personalized procedures and quality indicators.
\end{abstract}

Keywords: Ukraine; human development; higher education; quality assurance; semantic portal

\section{Introduction}

The challenges of societal development and change toward living long and healthy lives, being knowledgeable, having access to the resources for an acceptable standard of living, and participating in the life of the community are still actual in the new knowledge-based world economy (Streeten, 1994). The most fundamental dimension of development covers socioeconomic processes (Welzel et al., 2003) aimed at economic growth and an increase in social complexity which have been proven to be related to rising levels of education and increased access to information (Welzel et al., 2003).

The contribution of higher education (HE) is crucial: it is the main creator and disseminator of knowledge and social experience (Schleicher, 2006). Education can increase the human capital of the labor force, which increases labor productivity, and thus leads to a higher equilibrium level of output (Mankiw et al., 1992). According to UNESCO (2011), 
building effective education systems worldwide (from early childhood to the adult years and beyond) is extremely important for developing young generations into self-governing adults.

Numerous theories on human capital state that educational quality should be measured on the basis of obtained cognitive skills (not quantitative indicators of the societal enrollment in HE), which is the main driving force of economic, social, and human development. Analysis of the educational sectors in developing countries (Hanushek and Wößmann, 2007) proves this showing that educational quality is much worse than educational quantity. A strong relationship between the political power and academe influences the fact that quality assurance practices are used primarily for control purposes to suppress nontraditional institutions, programs, and teaching methods (Tomusk, 2000). Thus the road to improvement will involve major structural changes not resulting from merely adding resources but by also developing a strong quality assurance (QA) system aimed at enhancing the quality of teaching and research.

The chosen domain of the Ukrainian HE is very demonstrative. Ukrainian current HE system has changed little during post-soviet years and stays a state-driven, strongly centralized one (Tomusk, 2000; Temple et al., 2010; Harvey et al., 2010). External observers indicate poor performance of Ukrainian HE institutions (HEIs) in terms of innovative research, contributions to global knowledge, integration with international networks, mobility of faculty and student populations, links with business, and offering conditions for the formation of national elites that will stimulate good economic and political governance (Parusinski, 2010). HEIs`staff activities involve an extremely high volume of bureaucracy, where the officially reported "outcomes" and "achievements" are becoming more important than those actually achieved (e.g., the continuous process of reporting leaves little time to think about results and quality) (Harvey, 2011). Our data, which comprises numerous surveys of public opinion (Democratichni Iniciativi, 2011; UNITER/USAID, 2011), data from law enforcement authorities and State statistics service, HEIs staff and students' reviews, and many other sources, indicate a systemic corruption presence at all levels of the Ukrainian HE system, including in admissions to higher education institutions, in the academic process of teaching and learning, and in administering the newly introduced standardized test for high school graduates (Osipian, 2009). The state seems to encourage the institutionally based culture of corruption in higher education (Osipian, 2008).

An analysis of Ukrainian HE gives us a reason to consider that its poor performance is caused by distorted mechanisms of interaction between the society and the HE system. Important stakeholders are no longer motivated to participate in HE Quality Assurance (QA) 
processes. Request and pressures from society, the main consumer for HE services, are needed to stimulate the system players to evolve towards higher effectiveness and international standards. Fractured activities and efforts will not achieve any noticeable nationwide results until the whole of society evolves towards awareness of the importance of HE quality and elaborates a quality culture.

That is why the overall long-term objective would be the elaboration of this quality culture by supporting the interaction between HE members and interested stakeholders from the surrounding society. A new quality culture has to be introduced in order to augment the existing, state driven top-down approach that fails to meet the needs of the current economy.

We address these needs through an ICT solution that provides a digital ecosystem around a semantic portal, a platform for the delivery of socially verified data about Ukrainian HE. The portal helps establish a higher quality of communication between various HE stakeholders by providing them a forum to contribute HE quality information and to perform quality monitoring collaboratively.

\section{Methodology}

The objective of the research is to help increasing the societal influence on the HE system. The paper has a cross-disciplinary focus due to the following hypotheses: (a) human development in the country is related to the quality of education (b) a successful reform of the HE system in Ukraine can be essentially facilitated by a transparent bottom-up publicinitiated and public-driven activity (new, publicly visible, QA-processes, sharing of academic information with the public, social verification of available data and public assessment of reported academic achievements) rather than by strengthening the current top-down administrative control; and (c) such activity is actually a human development process with both an immediate effect (when a human evolves through active participation in social verification and public assessment processes) and a strategic effect (when the level of the societal development benefits from a qualitatively improved educational system). To address our objective and related hypotheses we partially rely on a qualitative research (analyzing documents and various opinions, making fieldwork, developing an understanding of the social and cultural contexts around our objective). However, we also attempted to provide more evidence on the validity of our hypotheses through quantitative research by providing an ITsupported experimental environment through which such public activity could be performed and with which the needed experimental evidence in favor of the hypotheses could be collected. Therefore our second objective is to design a digital ecosystem (Web based portal) 
for piloting public-driven QA processes. Taking into account challenging requirements, for such a portal, related to collaborative verification and assessment capabilities, this IT tool itself would require quite an innovative architecture and design methods. Therefore we are coming to the second objective of this paper, which is the design and implementation of a portal to serve: (a) as an instrument for quantitative research on the first objective; (b) as a pilot of possible IT-tool for public-driven QA processes in the Ukrainian educational system; and (c) as an instrument for human development. To report on this objective we will use some features of the design science research (Hevner, 2004). It relies on the behavioral-science paradigm predicting organizational and human phenomena surrounding information systems and the design-science paradigm seeking to create innovations through which information systems can be effectively and efficiently developed, managed and used.

The research questions to be addressed in the paper are the following:

1) How does the Ukrainian context influence requirements towards a successful QA system?

2) How can the power of public opinion concerning QA processes in HE be utilized?

3) How might IT support public-driven QA processes?

The following text of the paper will be organized according to the structure of a typical design research (Bider et al., 2013): in Section 3, we present a problem analysis and assess the relevance of the ICT solution for the revealed problems in Ukrainian HE; in Section 4, we define requirements and discuss a possible action plan and potential enablers towards a successful HE QA system; in Section 5, we present a semantic portal as an ecosystem for transparent and efficient QA activities (we present major requirements, architectural components, ontology, and component details such as the content management system with social verification and the reputation management, and personalized quality monitoring functionality); and we conclude in Section 6 by evaluating the results of the research.

\section{Related work}

In the context of human development, addressing problems by way of ICT solutions has become common practice. The openness, accessibility, and networking opportunities provided by the Internet and the Web give a powerful weapon for society in its struggle towards autonomous, deliberated behavior closely linked to socioeconomic growth. The field of education is one among many to reap the benefits of ICT products and services (Collis and Wende, 2002). It can improve the lives of many and particularly of people in transition 
economies. Assar et al. (2010) notes that ICTs have the potential to reduce barriers to education-for-many as they provide underserved and geographically and culturally disadvantaged societies with access to educational resources. For instance, the knowledge gap in low-income countries is proposed to be bridged by linking them with high-income countries by means of videoconferencing serving as an effective medium to facilitate trainings (Negash, 2010), mobility restrictions are overcome with the help of e-learning which enhances the educational process and provides an opportunity for continuous learning (Shraim \& Khlaif, 2010), people with disabilities are supported with software and hardware developed to deliver special educational needs (Nkansah \& Unwin, 2010).

Despite the fact that a vast amount of research already has been done by the world's scientific community on reforming education systems with help of ICTs, the main focus is rather on ICTs used as a part of the teaching and learning process while aspects of ICT support for education management as a means of improving educational quality are not widely studied. The quality issue is of utmost interest for Ukraine due to a need for structural changes in its HE and its QA system. As shown in the paper by Assar et al. (2010), the adoption of ICTs by education is an appropriate way to contribute to educational change (enabling structural reforms of education systems) in developing countries. ICTs can be used more as an instrument for reforming their inefficient or/and corrupted educational systems rather than merely as an instrument for smarter classrooms, enabling remote access, improved content management, or similar concerns. Deploying semantic tools and services across a field of linked data could be a way to address many current challenges of higher education management (Tiropanis, 2009). Murillo (2013) in her analysis shows how Web presence can reduce perceptions of corruption and recommends supporting and promoting the use of the Internet to supplement anti-corruption measures.

Similarly, ICTs should support the HEIss business processes to align decision-making with greater transparency, making the process quick and efficient. According to the report of the European University Association (2010) the overwhelming majority of European HEIs use information systems for monitoring their activities and collecting data about their performance but they typically do not share the collected information and do not seek feedback with the community. There is a wide-spread need for more valid and reliable information on academic quality as well as a need for public evidence that universities take self-regulation of academic standards seriously and that existing professional processes are designed to assure such quality (Dill and Soo, 2004). It is widely recognized that public trust (as a reflection of respect and confidence) is extremely important for the progress in HE. 
According to Leveille (2006) maintaining trust, providing well-conceived and meaningful performance indicators, and enabling data-driven decisions is essential for engaging all stakeholders in an HE accountability system. Elected officials, HE personnel, and its stakeholders must work in partnership with one another to carry out their respective roles in the national education system.

This can be enabled by Web-based tools, resources, services, and environments supporting new forms of public engagement and bringing an essential change to the way in which educational data is accumulated and processed. Using Web-empowered tools such as portals, a wider public can become involved in the quality assurance of education, making a means for control, open communication, and social contribution attainable. Portals are defined by Jafari and Sheehan (2003, p.69) as "a single integrated point for useful and comprehensive access to information, people and processes". They can be described as both personalized and customized user interfaces, providing users with access to both internal and external information.

Portals are now actively used for integration of information, services, and infrastructure across a seamless and easy-to-navigate Web interface in many companies and educational institutions. So called "campus portals" are providing access to university resources in a wide range of the American universities. Their functionality and architectural features are thoroughly discussed by Jafari and Sheehan (2003), Katz (2002), and Eisler (2001). The data warehousing and decision support tools of portals offer more advantages and opportunities to universities in terms of self-service, convenience, immediate access, flexibility and timeliness. M. Allayar's (2011) survey has shown that portals enable a significant devolution of organizational responsibilities, enhancements to service levels between central units and interdependent departments, and a reduction in the rates of growth of administrative cost by:

(1) Utilization of e-business technology.

(2) Integration and streamlining of information and services.

(3) Improvement in communication and collaboration via new communication channels such as automated alerts, email notifications, and SMSs.

(4) The facilitation of access to organizational information in a timely manner.

(5) Provisions for wider use of existing data and services.

(6) Improvement of services to students, academics and staff.

(7) Creation of a framework for managing the organization information environment. 
Richard Katz (2002) also points to so called "customer relationship management" tied to the portal concept, referring to it as a feature developed to enable institutions to create communities and sustain relationships between the institution and its stakeholders, which is essential for our needs.

The widely discussed semantic information portal (Maedche et al., 2003, Lee et al., 2010), is a community information portal that exploits Semantic Web standards to improve structure, extensibility, customization, and sustainability (Reynolds et al., 2004).

There are a number of advantages of using Semantic Web standards for information portal design over the traditional approach (Reynolds et al., 2004). The most important are:

(1) Information is semi-structured and extensible, allowing for bottom-up evolution and decentralized updates.

(2) Multidimensional search is possible.

(3) Information structure is machine accessible directly.

(4) The information structure is flexible and can be extended externally.

(5) Portal content is stored and managed by a decentralized web of suppliers, representing both organizations and individuals.

(6) Providers publish data in a reusable form.

The kernel of the system, developed according to the semantic approach, is formed by an ontology-driven knowledge base on top of two Semantic Web formats: RDF (the Resource Description Framework), which provides a standard "triplet-based" model for data interchange on the Web that enables setting relationships between different pieces of the information (Klyne et al., 2004), and OWL (the Web Ontology Language), which supports explicit representation of the domain ontologies used to classify and structure the items (McGuinness et al., 2004).

\section{QA Concept for Ukraine}

\subsection{The essence of the Ukrainian HE problems}

Ukrainian HE is still managed by a highly centralized real-time administrative command system. A state-owned HEI is totally controlled and managed by the Ministry of Education and Science of Ukraine (or in some cases by another ministry). Its personnel behave reactively following orders from above, orders which have a short-term visible effect for an HEI. Unlike an independent unit, which is concerned with demonstrating maximal 
effectiveness and appearing proactive by nature (in setting long-term goals and implementing an evolutionary strategy), a state-owned HEI that is controlled from outside cannot follow any internally developed strategy, no matter how brilliant and timely it is. This reactive mindset is fostered through all the HEIs` levels (from the ministry via the rector up to a teacher) causing a lack of academic freedom and creativeness. In addition, there is usually no link between an HEI's real performance level and the amount of resources obtained from the State, causing a lack of motivation to evolve, to perform unbiased self-evaluation, or any other QA procedures aiming at real quality improvements.

Experts from the International Bank for Reconstruction and Development (Hanushek and Wößmann, 2007) suggest that three institutional features of a successful educational system be considered by developing countries in their attempt to reform:

(1) Choice and competition.

(2) Decentralization and autonomy.

(3) Accountability for outcomes.

A key solution can be indeed seen in the potential for the self-organization of the HE system, restricted only by general rules and high-level policies. Despite the need for change, giving immediate autonomy to HEIs can be harmful considering that there is currently no quality culture elaborated by the Ukrainian society, and important stakeholders (e.g., students, industry, external organizations, international experts) have no legal means and (what is more important) no willingness to influence decisions within the QA system (Gryshko, 2012).

Therefore we propose to divide the HE QA system development into two stages:

(1) Creation of the background for the responsible societal influence on the quality of HE by the voluntary involvement of a wide range of stakeholders in the QA process.

(2) Gradual elaboration of the concept of national HE quality according to the challenges of the global economy and to social demands.

\subsection{The first stage of the $\mathrm{HE} Q A$ system development}

The first and the most urgent need, which is a basis for a successful HE QA system development and further sustainable adoption and exploitation, is strengthening communication between HE players and the society (see, Van der Wende, 2000; Stensaker, 2003; Hoecht, 2006). One possible way to push the process forward is to ensure the transparency of the various levels of the national HE system and give a channel (an open ICTdriven platform) for direct communication between the society and HEIs thereby providing 
(a) public access to the information about existing educational system, players, and processes within it, as well as to the information about orders and transactions, achievements and their impact; (b) a means of the public influence and control over the truthfulness of the published information by social verification mechanisms; (c) tools for the versatile processing of the raw information to get necessary analytics from the information in the form of statistics, rankings and other assessment reports; and also (d) a means for getting feedback on the published information from the society.

The first stage will start the process of building a transparent trusted environment around the evidence of the importance of HE quality.

\subsection{The second stage of the HE QA system development}

After the means of involving the public in the HE QA process is nationally introduced, the second stage starts. It aims at accumulating the social demands and incorporating them into the practices of the HEI and promoting the best practices among the Ukrainian HE players to lay ground for the development of QA procedures that are based on credible, transparent, and relevant sources of information and explainable decision-making.

We recommend the following multi-layered structure of the stage:

(1) The creation of a national society-driven quality concept based on possible utilization of the best European practices.

(2) The development of a national culture of the unique QA for an HEI with respect to both the HEI`s specific context and the national quality concept.

(3) The development of national and institutional transparent and reliable QA procedures for HEI evolution aimed at achieving the chosen quality standards.

\section{National quality concept}

Though the importance of QA as a powerful driver of change in higher education is widely recognized, a wide range of experts in Ukraine cannot name either common requirements to the HE quality or a widely accepted definition of the corresponding quality concept. The national QA system is based on the distorted and outdated "value system" (i.e., quality indicators and their relative importance) imposed by governmental demands but not societal ones (Harvey et al., 2010). This therefore results in the inappropriate fitness function (i.e., a formal instrument used by many disciplines to assess and control an evolution process) of monitoring the evolution of the educational system towards better quality objectives. Data about educational processes declared by HEIs in various reports are redundant, irrelevant, and 
become outdated quickly. Moreover their statistical nature cannot reflect the real quality of the HE services and achievements, prompting an urgent need for a national value system as a set of general properties characterizing HE quality. To ensure relevance, we suggest that its main preconditions include:

(1) The denial of the numeric statistical properties used in the current quality evaluation procedures and, rather, focusing on a set of the properties showing effectiveness of the HE through its impact on real social and academic progress.

(2) The formation of new quality indicators from the perspective that the main task of HE is knowledge creation, innovation, and transfer facilitated by the balanced interaction of three vertices of the knowledge triangle (education, research and innovation).

(3) A revamping of quality criteria sets and standards for QA with attention to best international experience and practices (see European Association, 2009 for a good default value system that allows for customization according to the current national needs and social demands).

(4) An attention to ensuring both the inclusion of and a means to motivate important stakeholders to participate fully in the development of a national value system.

\section{Context-sensitive $Q A$}

We agree with Weisberg (1974):““.. I would contend that analysts frequently should not seek a single measure and will never find a perfect measure. ... It is time to stop acting embarrassed about the supposed surplus of measures and instead make fullest possible use of their diversity ..." Each HEI should develop its own unique evolution strategy with respect to both the national value system reflecting the main societal demands and particularity of the HEI s specialty. The developed strategy is a value system model consisting of a pertinent set of HE quality properties weighted by their relative importance.

Developed models should be fully accessible for all stakeholders and HEIs to view in the interest of improving their own models. This should increase the societal trust in those HE players who are demonstrating good QA performance and will lead to the creation of groups consisting of HE players with common interests and opinions regarding QA.

To find its own optimal strategy, an HEI may apply solutions similar to optimization problems, using techniques inspired by natural evolution - partial artificial selection mechanisms and optimal solution search algorithms that use inheritance, mutation, selection, and crossover of the value systems. 


\section{Trusted QA procedures}

A complete and consistent QA system according to its traditional meaning is absent in Ukrainian HE. It is partly implemented, in form of quality monitoring procedures which are performed on the basis of the same criteria set for all types of HEIs. There are no procedures for strategy planning of the HEIs evolution as well as no real quality objectives to achieve, maintain or enhance. The reason is seen in the fact that the actual goals of what is called, in Ukraine, a QA system are not about helping the HE players to evolve towards higher quality but rather to control, to apply pressure on, and to punish those who do not fit the given criteria set. Existing QA procedures are transparent neither for the actual participants nor for the external observers (society, industry, national, and international experts). There is no transparency on how the observation (endless monitoring) of "quality" leads to decision making and action. With all the above, there is a redundancy of the QA players. Their uncoordinated activities and numerous procedures results in a degeneration of the corresponding procedures and a decrease of the HEIs motivation to take part in them. Not all the State commitments regarding HE and science are implemented as reported. All the individual and organizational QA players are dependent on the State authorities (for both their existence and funding) and therefore their trustworthiness is questioned due to the evident bias in their decisions, actions, and reports.

QA procedures may vary for HEIs particularly due to their diverse specialties. Our study (Towards Trust in Quality Assurance Systems, 2012) showed that all the procedures should be widely accessible and open, to ensure achieving, maintaining or enhancing quality by complying with the following axiomatic conditions common for successful HE systems:

(1) High level qualification of the academic staff.

(2) Motivation of all the parties to take an active role in improving the quality of HE.

(3) Transparency of information on all levels of the HE system (including QA procedures).

(4) Sufficient amounts of resources (financial, technical or service-based) and their distribution, which would reasonably correlate with the achieved quality.

(5) Flexibility of the educational content.

(6) Mobility of academic staff and students.

(7) Academic freedom, autonomy and independence.

The monitoring procedures should be performed by an independent QA body evaluating an HEI`s (or other academic resources) performance based on the national quality 
concept and the HEI's value system. The QA agency should be a member of ENQA: the European Association for Quality Assurance in Higher Education (www.enqa.eu) to support its independent and trusted status and gain access to important ENQA services, such as a forum for communication with European quality assurance experts for advisory support. Participation of international experts is essential.

A fitness function for QA performance monitoring should be chosen to determine how well the evaluated goal corresponds to its associated predefined criteria, including the possibility to evaluate the QA procedures undertaken to achieve the goal.

Results of the evaluation of the QA procedures should be transparent and should influence resource allocation (meaning all type of resources: financial, technical, and human resources, awards, degrees, etc.) which should be distributed using common transparent rules (both regulatory and legislative) for all the HE players.

\section{The National Quality Assurance Portal}

\subsection{A semantic portal as a tool for national reform}

One possible method of achieving the aforementioned reform in HE can be accomplished in the use of appropriate ICTs, for example, use of a semantic portal as a social barometer for academic quality. The National Quality Assurance Portal (hereinafter the Portal) provides a set of solutions that enable, support, and automate the activities, information flows, and transactions within the ecosystem of individuals, HEI staff, and QA organization personnel, who are exchanging information for Ukrainian HE QA. The open portal environment and personalized QA approach makes schemes (value systems, QA procedures, strategies) used by different QA players transparent and shareable (Terziyan, 2011).

The "semantic" aspect of the Portal is considered an important one. Taking into account that the Portal must work with constantly updated data, driven by constantly evolving knowledge and policies, being constantly updated by new information providers and with new features in the data in real time, we had to design it in accordance with to the Open World Assumption. This assumption (in formal logic terms) reflects the monotonic nature of the first-order logic, in other words, adding new information never falsifies previous conclusions. The Semantic Web standards and technologies are created to support architectures and data and knowledge collections, and to provide reasoning support for applications that follow the Open World Assumption. Another reason for semantics is related to the potentially heterogeneous nature of data sources and Web-services connected with the Portal, which may 
provide a variety of structures for the data and poly-semantics to the concepts. To enable seamless integration of the data and the interoperability of various services, there is a need for the ontology-driven (semantic) architecture of the Portal. Among other reasons to have a bold semantic aspect in the architecture, one can mention also the need for automated processing of information using various standard tools as well as in-house analytics. Automated processing of linked open data is known to be one of the reasons for the need for semantic technology.

The Portal evolved as follows: the first version was a proof-of-concept pilot (20072010), developed for the centralized accumulation of information regarding Ukrainian academic resources developed mostly under the requirements of the Ministry of Education and Science of Ukraine, after which a testing period transformed the pilot into the second (current) version. The latter one (in accordance with the new QA concept) is an outcome of the on-going development effort performed according to the TEMPUS project specifications (http://dovira.eu) for the creation of the transparent trustworthy ICT HE environment to be maintained by all HE players and stakeholders themselves (http://portal.dovira.eu). It was assumed that if real autonomy of the QA agencies is not reachable in Ukraine in the immediate future, the portal can be considered as an alternative, credible, and independent ICT-driven "QA agency” available for the Ukrainian academic society and beyond.

\subsection{The portal operating side}

The Portal was developed on the Java Enterprise Edition platform and runs on the JBoss Application Server (Figure 1). Java Server Pages technology is used for the development of dynamically generated web pages. The Java application programming interface for XML Web Services and servlet technologies enable remote interaction of Web-services. The ontologydriven kernel, served by the OpenLink Virtuoso server, is designed as an ontological knowledge base, and semantic API for handling ontologies for open and flexible storage and processing of information. 


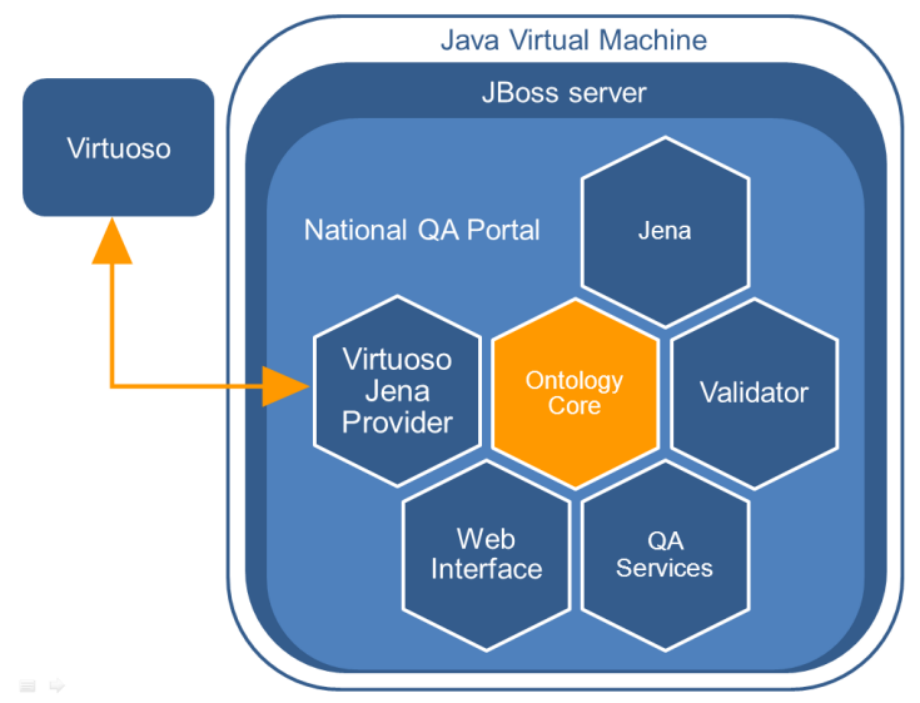

Figure 1. Components of the Portal's operational environment

To enhance the Portal's social function and to avoid the total information control by some dependent party, the system is built based on the social network principles: users are main contributors, controllers, and beneficiaries of the Portal's content and facilities. The Portal provides a technical means for:

(1) Collaborative registering of facts about national academic resources (heterogeneous by their nature, comprise players and processes within HE, orders and transactions, best practices, achievements and their impact, and etc.). A user of the Portal starts playing the role of content-manager after the registration and creation of his/her semipublic profile. In other words, his/her contributions are visible to others in an attributed (not anonymous) nature, but are not directly editable by all and are not directly reflected in a collaborative space. Rather, the public space evolves collaboratively based on the most trusted information from users` private spaces using a social verification instrument.

(2) Giving wide public access for browsing of the published information to all visitors (both registered and unregistered in the Portal).

(3) Gathering users` feedback on the accuracy of published information via social verification methods (e.g., referencing, cross-validation, voting, challenging, forums).

(4) Encouraging users' contributions and managing users' reputation.

(5) Avoiding information redundancy by recognition and merging of similar resources.

(6) Providing services supporting HE QA procedure simulation (e.g., quality evaluation by ranking) by processing the facts registered in the Portal and information obtained from trusted external sources. 
(7) Offering flexible user-dependent customization of QA procedures by creating userspecific value systems including multicontextual HE quality indicators weighted by their user-determined relative importance.

(8) Enabling access to collection, analysis and distribution of QA data obtained through anonymous or targeted queries or surveys, or by importing external statistical data.

\subsection{Knowledge and (Meta)Data Collection in the Portal}

The knowledge base is driven by two ontologies: Service ontology and Domain ontology. Service ontology contains auxiliary classes and properties for system business logic, resources registration support, business analytics, QA procedures simulation, and for other similar purposes. It is designed to serve as a domain independent structure, flexible enough for interaction with ontologies describing any possible domain (not restricted by $\mathrm{HE}$ as in our case) within a resource management system that supports quality monitoring. The ontology provides a scheme based on relation within four main classes:

(1) Resource. Any item of the domain registered in the Portal.

(2) ValuableResource, a resource deemed useful for quality evaluation.

(3) Beneficiary, an organization or individual whose performance assessment depends on the valuable resources that they own.

(4) Achievement, an entity indicating a fact of the ownership of a valuable resource by a beneficiary.

The ontology model is adapted to collect a history of resource changes via instances of the HistoryItem class linked to a resource property value.

Domain ontology lays HE QA domain-specific entities over the Service ontology. It comprises (a) the core layer, which specifies concepts and properties related to the knowledge triangle (education, research and innovation) used for HE QA; (b) the customized layer, which every organization can flexibly adapt to a local context or every user can adapt to his/her preferences; (c) the system of values, which defines weights for various quality indicators in various contexts; and (d) the QA processes, formally specified internal or crossorganizational processes that enable QA execution monitoring (Terziyan and Kaykova, 2012).

Figure 2 demonstrates a possible way of interaction between the Service and the Domain ontologies. Research papers, theses, patents, training courses and other documented achievements are considered as valuable resources in the HE domain. All semantic annotations of registered resources of the aforementioned types are stored in a common pool 
within the system, corresponding to the core layer of the ontology. Any educational organization or a person from academic society (defined as a beneficiary in the Service ontology) can be linked with a specific valuable resource by marking it as a personal/organizational achievement. Thus a beneficiary collects all his/her/its achievements linking them to his/her/its profile under the strict control of portal users engaged in social verification processes. Each achievement adds potential points to the evaluation by the beneficiary. The amount of the points depends on the impact of the resource weighted by a respected third party (e.g., Google Scholar web-service) and the evaluator`s system of values defining the importance of each type of achievements and comprising the customized layer of the ontology.

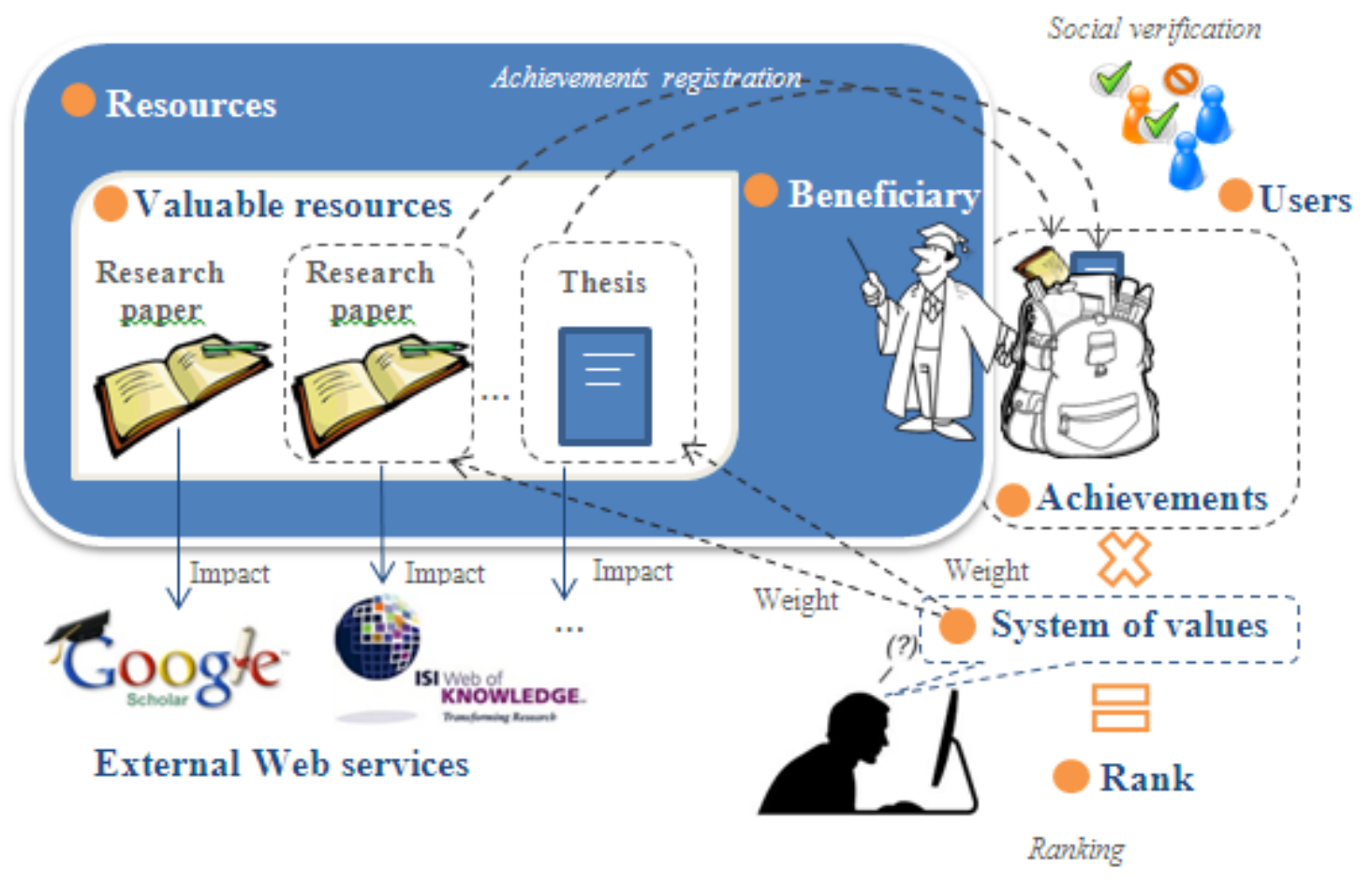

Figure 2. General workflow of the portal information

The stored knowledge is:

(1) Intelligent due to utilization of a self-organized semantic storage (see Muhleisen et al., 2011) which enables QA processes to be self-descriptive and self-managed instances.

(2) Cross-validated, in that automatic updates are partly enabled by the Service-Oriented Architecture. For example, the impact of the educational and scientific resources is taken from external Web-based sources, such as ISI Web of Knowledge, Scopus or Google Scholar.

(3) Self-explanatory, meaning if provides supporting, detailed explanation of every calculated or inferred value of every quality indicator used in QA activities. 
(4) Context-aware, due to utilization of knowledge about context (local, regional, national, international) for more grounded evaluations.

\subsection{Quality evaluation services provided by the Portal}

Several quality assurance options are supported by the Portal services, for example, comparative evaluation (ranking) of the registered resources in which the end value of the evaluated resource would be its position in a rank list of resources of the same type. Each ranking is performed on-the-fly from a user`s request, and ranking results are stored in the knowledge base for possible reuse. The Portal allows creating and applying different value systems in the form of flexible multidimensional and multicontextual HE quality indicators weighted by their importance to the ranking query. Thus each user can evaluate the relational quality of some HE resources from different points of view (representing, for instance, different stakeholders or different world ranking systems). Such a procedure is based on the information regarding the relative importance of different achievements which are taken into account when evaluating the "owner" of the achievements registered directly in the Portal or imported from trusted sources.

A vector of numbers from 0 to 1 shows the importance of the achievement to the person creating the rank. The interface for creating and maintaining a personal system of values is shown in Figure 3.

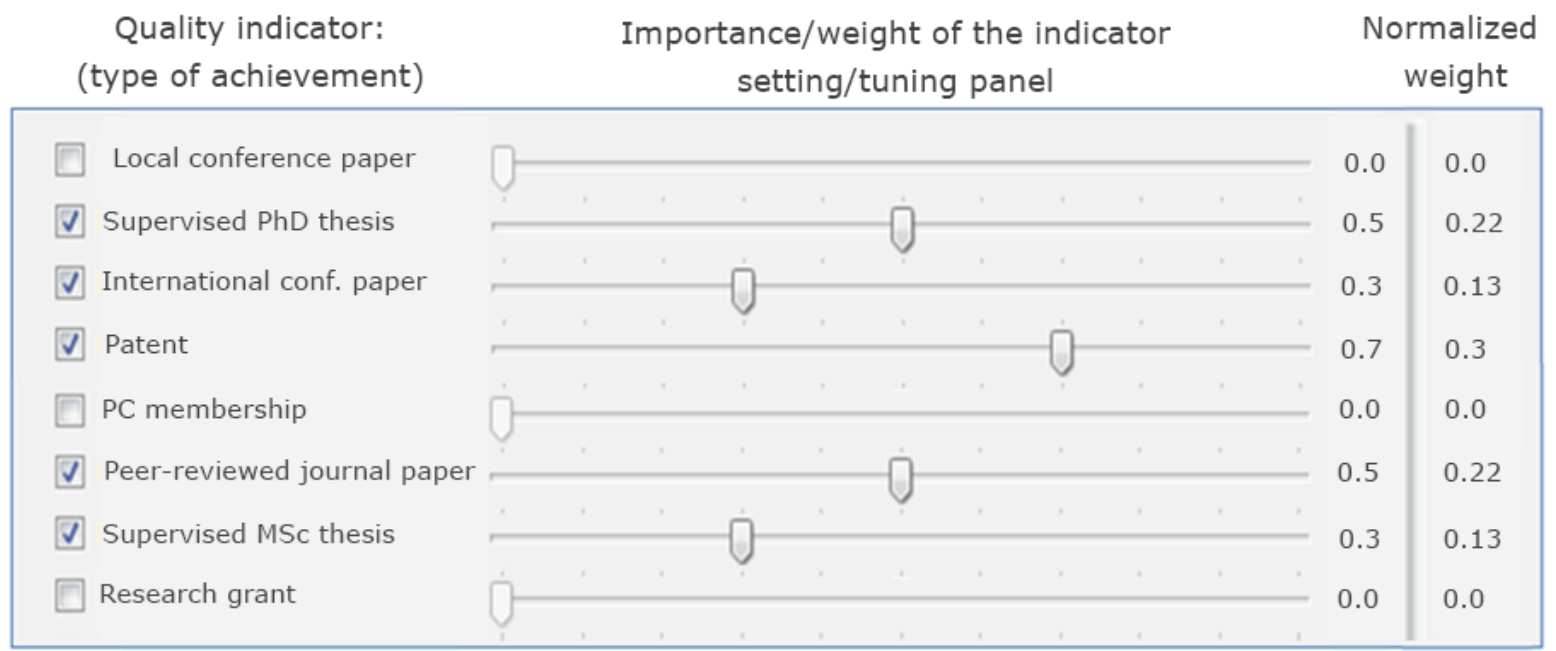

Figure 3. The Portal interface for creating a personal system of values

Analysis of the collected data will help an HEI to choose an appropriate evolution strategy and will give a clear picture of the HE system state to all interested parties.

The preparatory phase for the ranking procedure consists of the following steps: 
(1) Selection of the set (class) of instances to be ranked using the ranking interface by either enumerating needed instances or by the use of logical expressions and constraints (filters) applied to the classes of instances in the Ontology.

(2) Choice of ranking system, a step in which a user can choose from the list of the supported rank calculation systems, use the search utility to search for the supported ranking system by name, or use the advanced search choosing from a set of filters.

(3) Creation (or selection if created earlier) of his/her/its system of values.

(4) Creation of a schedule for automatic ranking queries and choosing the means of obtaining reports (via the Portal interface on demand or by receiving ranking report by e-mail).

The ranking procedure itself goes as follows: each entity (e.g., a University) obtains a numeric value (cost) as a result of evaluation of all its achievements (e.g., papers published by the university staff) calculated by:

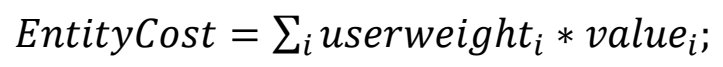

where value $_{i}$ corresponds to a value of all achievements of the same type (i) and userweight is a user-defined weight for the type in the chosen RankingSystem for the current evaluation request.

The value for all achievements of any one type is calculated as:

$$
\text { value }_{i}=\sum_{j} \text { resourceweight }_{\text {type }} * \text { impact }_{j}
$$

where $i$ is an achievement instance in question, resourceweight $t_{\text {type }}$ is a default value for the type of the achievement being inversely proportional to the number of the achievements of this type (rarer achievements are more valuable) and impact $_{j}$ is an impact of the achievement calculated differently for achievements of various types (e.g., scientific papers have citation-based metrics for impact calculation).

Resource allocation in HE should be based on the results of the quality evaluation procedure. For example, $N$ is a total amount of resources to share between the universities, and each university's share is based on its achievements (as shown in Figure 4). A responsible body (e.g., the Ministry of education) appoints some weights (weight $t_{i}$ ) to each type of the university achievement according to the national strategy or due to other motivations. Each group of national achievements will be associated with a concrete amount of granted 
resources and is calculated as weight $_{i}{ }^{*} N$. Calculation of the amount of resources required for an achievement of a certain type can take into account its impact. The share for the evaluated entity (e.g., a university) is simply a sum of resources granted for the associated achievements.

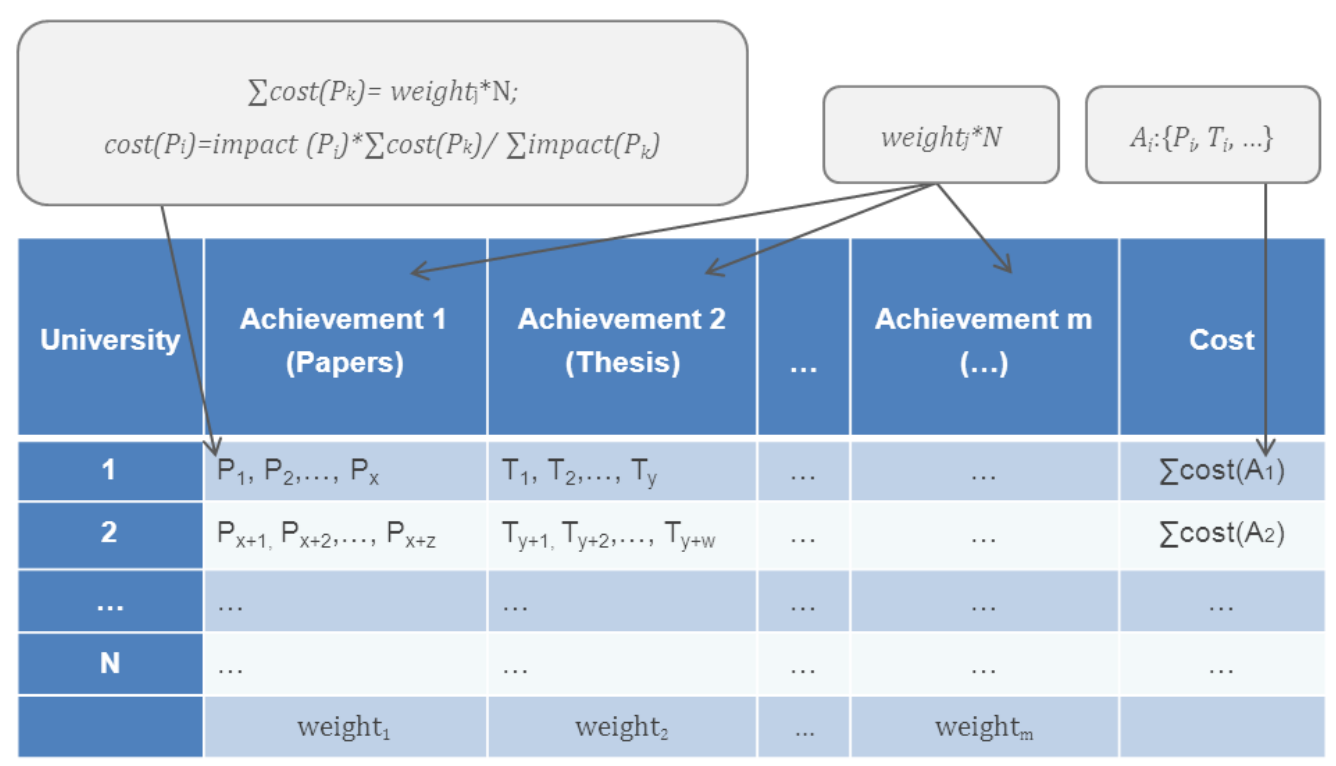

Figure 4. Schema of the resource allocation based on ranking

\subsection{Social verification of the information registered at the Portal}

Social verification is an aspect of the Portal mechanisms that involves the wider community in the creation and confirmation of information correction on the trustworthy shared HE space. Utilizing social verification we attempt to harness the benefits of combining the advantages of semantic (Web 3.0) and social (Web 2.0) computing, as discussed in (Ankolekar et al., 2007).

The absence of trust in the Ukrainian HE causes a need in the wider public for the opportunity to contribute to information accumulated in the Portal. On the other hand, currently used social network principles make different hostile manipulations of the information possible. That is why the consistency and accuracy of the Portal information and an unbiased form of decision-making is ensured by joint moderation (see Figure 5). 


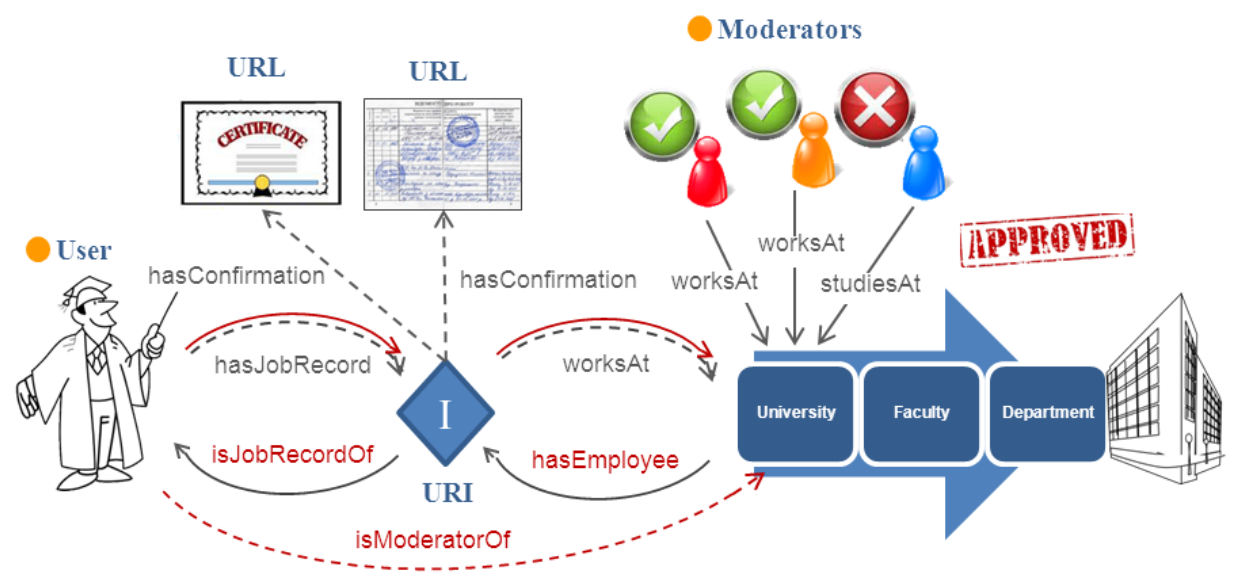

Figure 5. Scheme of social verification processes

Each resource has a number of moderators - all users linked to the resource by one of many types of relations. Thus a university, for instance, is moderated by all its students, academic staff and everybody else who has created a verified link between his/her profile and the university. The creator of the resource automatically becomes its first moderator and is selected to be a moderator of all related resources. In practice, a moderator makes decisions about the correctness of the moderated resource`s properties values. For an object type property, the moderators should confirm that a resource set by a user as a property value is indeed related to the moderated resource. For example, a user, indicating a university as his/her place of work, sets the value of the property worksAt equal to the university resource, which causes an automatic request to be sent to all moderators of the chosen university to confirm the fact. Confirmation is achieved by the creation of the inverse link: setting the correspondent object of the inverse ontology property (hasEmployee). The success of the query will require the weighted majority of the moderators' votes. A weight of the user`s vote is related to his/her reputation rank, which will evolve depending on his/her actions observed within the Portal.

To describe correctly a user`s employment and study history we gather all pieces of information about a certain user`s activity in special job records, study records, and so on using ancillary instances I (see figure 5) for this in ontology. The link between a user and a resource of a different type is created via these records.

A moderator of a resource or of the system can also initiate the merging of a moderated resource with other resources (originally registered as unique but currently suspected to be the same as an existing resource) by sending a corresponding ("co-reference resolution") request to all the moderators of each resource. 
Participating in voting or any other verification activities is absolutely voluntary and one can always remove himself/herself from the list of the possible voters for a specific request while still remaining a moderator of the resource.

The Portal gives a possibility to state some facts proving the correctness of the registered information by adding links between a resource and external Web pages containing confirming documents, for example, certificates, and other images and scans.

\section{Reputation management in the Portal}

To encourage a users` helpful involvement in the processes of the educational environment in the Portal, a reputation management system is developed. Advanced capabilities offered by the Web 2.0 to its users easily connect people together, forming big virtual communities collaborating in various professional environments. To manage overwhelming size of such communities, various reputation mechanisms were elaborated to support members with information about partner's trustworthiness with respect to their observed behavior and contributions. Skopik et al. (2009) made a thorough review of these mechanisms. For our study we have chosen the multiplicative mechanism for reputation ranking and refinement. The advantage of it is that a more reputed member of the community has more power in collaborative voting but at the same time has a greater responsibility (punishment) for a mistake.

Each user has a portal reputation in form of a number between 0 and $\infty$. This value does not indicate the user`s academic or scientific quality obtained via a formal quality evaluation procedure but, rather, it grants distinguished voting rights within the Portal, awarding reputation rank increases for active users (contributors and moderators) and applying certain "punishments" (penalties or ranks decrease) to those users who do not take active part in the content creation and social verification processes. Reputation management is related to a user`s behavior in the Portal while formal quality evaluation is based on a user`s academic and scientific achievements.

The value of the reward or the penalty for a particular action performed by the user depends on the action taken and the current reputation of a user. During the content creation, verification, and utilization process, there are several possible user actions, which either positively or negatively influence his/her reputation rank (see Table 1 for a ranked list of user actions ordered according to the magnitude of reward/punishment he/she will receive from the reputation system). 


\begin{tabular}{|l|c|l|c|}
\hline \multicolumn{1}{|c|}{ “Good” behavior (increases rank) } & W & “Bad” behavior (reduces rank) & P \\
\hline Replying to a request & 1.05 & Abstaining from voting & 0.95 \\
\hline Querying the Portal functions & 1.1 & Not replying to vote requests & 0.9 \\
\hline Editing his/her own resource & 1.15 & $\begin{array}{l}\text { Voting, unsupported by the } \\
\text { majority }\end{array}$ & 0.85 \\
\hline Voting, supported by the majority & 1.2 & $\begin{array}{l}\text { Proposing, not supported by the } \\
\text { majority }\end{array}$ & 0.8 \\
\hline Creating a new resource & 1.25 & $\begin{array}{l}\text { Challenging, not supported by the } \\
\text { majority }\end{array}$ & 0.7 \\
\hline Proposing, supported by the majority & 1.3 & $\begin{array}{l}\text { Requesting a merge, not supported } \\
\text { by the majority }\end{array}$ & 0.6 \\
\hline Challenging, supported by the majority & 1.4 & $\begin{array}{l}\text { Being challenged, supported by the } \\
\text { majority }\end{array}$ & 0.5 \\
\hline $\begin{array}{l}\text { Requesting a merge, supported by the } \\
\text { majority }\end{array}$ & 1.5 & SPAM or Abuse & 0.3 \\
\hline
\end{tabular}

Table.1. Multiplicative rewards (W) and punishments $(\mathrm{P})$ within the reputation management system.

New users are assigned an initial reputation (or reputational rank) of 1 . The positive reputational rank update (see the left side of the Table 1) for the "good" user action $i$ is accomplished by multiplying his/her current reputation to the number $W_{i}$, which is greater than 1. The negative reputational rank update (see the right side of the Table 1) for the "bad" user action $j$ is accomplished by multiplying the current reputation to the number $P_{j}$, which is less than 1 . The concrete magnitudes (vectors $\mathrm{W}$ and $\mathrm{P}$ ) of these rewards/punishments are control parameters which are defined during initial configuration when the Portal is installed.

\subsection{Piloting the Portal}

The Portal was launched and piloted based on information collected from four Ukrainian universities constituting a consortium representing different types of HEIs including Kharkiv National University of Radioelectronics (KNURE), one of the oldest Universities of Technology in Ukraine with over 12,000 students and 30 scientific centers; Yuriy Fedkovych Chernivtsi National University (CHNU), a classical university and a significant geopolitical higher educational and scientific center that is internationally active and located in SouthWestern Ukraine; National Academy of Managing Personnel of Culture and Art (NAMPCA) in the capital area; and Ukrainian Catholic University (UCU) representing the Western region of Ukraine and the private sector of HE. During half of a year about 500,000 resources were registered by the users and about 15 million of knowledge triples were added to the knowledge base. The Portal was deployed on a dual-processor server ( 8 cores on each 
processor) with an operational memory of $128 \mathrm{~GB}$, which enabled sufficient speed for a system of this type ( 3 seconds for loading information about a resource and less than 10 minutes for caching information on initial startup).

The process of HE reforming, based on the principles of electronic democracy and responsible contribution of different stakeholders, may take a decade until it has impact nationwide and the effect becomes truly visible to external observers. Though initial outcomes of the introduction of the developed technology related to the transparent bottom-up public-initiated and public-driven activity are already visible:

(1) Academic and administrative staff participating in the pilot evolved towards a better understanding of the HE quality concept and European standards of the quality through active participation in social verification and assessment processes. They gained skills in ICT utilization for transparent resource management for selfdevelopment and institutional strategic planning (Gryshko, 2012).

(2) Universities participating in the pilot were reorganized to guarantee quality assurance on the institutional level. KNURE started the procedure of establishing an office of strategic planning and quality evaluation headed by a vice rector in order to elaborate quality policy and strategy of QA and to establish a quality management system aimed at continuous quality improvement. UKU appointed a person responsible for introducing QA (bounded by official duties). It introduced an updated concept of strategic university development, launched a new workflow system and an electronic system that collects students' evaluation of the quality of education, and developed new mechanisms of the professional growth for teachers` promotion.

(3) New QA procedures that utilize the advantages of electronic democracy, supported by the proposed technology, were developed to be adopted on an institutional level including the management of academic recruitment, the management of academic staff assessment, the management of academic staff motivation, the management of academic staff's life-long-learning, resource allocation management, innovation management, educational (tangible) asset management, student feedback management, academic networking management, and the management of internationalization.

(4) Communication with state authorities and society has increased. Experts from the consortium entered the working group of the Committee (Parliament Committee on Education and Science, 2013). Amendments to a version of the draft law on higher education concerning the elaboration of a quality culture: a necessity for the public 
reporting of academic achievements, transparency of QA procedures and evaluation based on internationally recognized quality criteria were proposed to the Parliament Committee on Science and Education. New versions of financial regulations were also sent for approval to the Cabinet of Ministers of Ukraine.

(5) A new set of quality criteria based on the best European experience was developed by a QA body - the State Educational Inspection Unit - for HEI's quality monitoring (State Educational Inspection Unit, 2013).

(6) Public influence on HE quality was significantly strengthened by student and nongovernmental organization representatives who joined the community of Portal users.

\section{Discussion}

Currently the Portal provides a platform on which different communities of interest (around HE) can be formed. The most obvious community of interest will be formed from academics that have genuine interest in improving the quality and learning from peers, without any external motivation (except peer recognition ), "the academic elite". Each nation creates its own specific academic elite with its unique advantages. If the culture is closed (no pressure to improve), it leads to degeneracy. The national academic communities of developing countries are exposed to this danger due to relative isolation from the international scientific community as well as from the surrounding society.

The academic elite and the surrounding society should interpenetrate (despite differences in their value systems). Within international science, community interaction with national academic cultures is accomplished by way of an old tradition that arose due to the exposure of international (and intercultural) assessment of achievements. A similar culture of interaction is needed also between academic communities and the surrounding societies. While it is impossible to change the culture directly, it is possible to influence it. If the academic elite are chosen by the direct combination of academic achievements and the value systems of the society, then the academic culture is formed indirectly (implicitly). Moreover, culture is usually a result of the evolution of the value system of the whole society; it starts to change as a result of the interpenetration of academic elites. Mutual enriching of cultures leads to strengthening of the factors of the development not only of education but of a nation as whole. The mechanism of such mutual aid leads to co-evolution. Two main driving mechanisms for co-evolution of academic and society cultures are: raising awareness of the influence of academic achievements in socioeconomic and human development, and the promotion of a culture of social verification of academic achievements. In the future, use of 
the Portal should address the aforementioned challenges as a result of the interest raised by its dissemination and due to it enabling the society (different stakeholder communities) to participate in assessing higher education and verifying the relevance of the results.

\section{Conclusions}

In this study we assumed that a reform of the HE system can be facilitated by a transparent bottom-up public-initiated and public-driven activity, which itself can be qualified as a human development process. To argument this hypothesis we addressed several research questions listed in the Methodology section and provided some experimental evidence for the validity of our findings by designing and testing the pilot.

We have shown that the quality assurance as a complex of activities enhancing effectiveness of an educational system is an important enabler of human empowerment. We pointed out that the greatest damage to the quality of higher education in many developing Post-Soviet countries, including Ukraine, is due to the fact that this implication is not evident to the governmental officials who seem to encourage the institutionally based culture of corruption in higher education and use QA practices primarily for control and punishment purposes. The main beneficiary of educational services quality - society - is left out of the quality monitoring and management. In our research we show that taking part in public QA activity would be a shortcut to participatory development by enhancing human autonomy and strengthening the conviction that an individual and the overall society can take responsibility for state decision-making. Therefore we are confident that QA in HE is an important human development process.

Such an approach to transparent public-driven QA is rather new and experimental, and, based on our knowledge, has never before been implemented in any country. This may be due to the fact that developed countries have a strong quality culture that has been elaborated by their societies for centuries, while Ukrainian society traditionally has followed an administrative centralized control. The use of an adopted traditional experience from other educational systems can be an option only after elaboration of the corresponding quality culture in Ukrainian society, meaning responsible societal positioning towards the quality of HE by the voluntary involvement of a wide range of stakeholders into QA.

To enforce the power of public opinion concerning QA processes in HE, we propose the mechanisms of social verification and assessment of reported academic achievements based on flexible multidimensional and multicontextual quality indicators to enable 
management of constantly variable contexts (caused by political, economic, or other reasons). This is a forum for appropriate QA strategy development and responsible decision making.

The pilot study shows that the roadmap towards the new QA culture should utilize the mechanisms of electronic democracy applied on top of the Internet, Social Web and related technologies with the goal of enabling collaborative content creation and management, verification and assessment, and utilizing an open Portal environment for these purposes. The Portal guarantees transparency of the existing and future QA processes, social awareness of the QA system, and enables wide public involvement in the process, including international QA experts' engagement and transparent sharing of their experience. It accelerates the longterm process of quality culture development and adoption.

Developed mechanisms, such as content storing and verifying, conflicts resolving, reputation management, private spaces management, the use of personal systems of values, and flexible assessment and ranking described in the paper, address architectural and design challenges related to the Portal social facilities. The semantic approach ensures decentralization of information updates, effective information management due to use of semi-structured semantically apprehended data and the customization of portal functions such as user evaluations of HE resources based on the users`own systems of values.

\section{Acknowledgements:}

This research has been supported in part by the TEMPUS grant 516935-TEMPUS-1-2011-1FITEMPUS-SMGR for the project TRUST: “Towards Trust in Quality Assurance Systems”. The authors appreciate the collaboration and support it has received from the international team of this project. We are grateful to the anonymous reviewers for their valuable comments, which helped to essentially improve the paper.

\section{References:}

Altayar, M. (2011). An Investigation into the Adoption, Implementation and Utilisation of Campus Portals: A Comparative Case Study of Saudi and UK Universities (PhD Thesis). De Montfort University, Leicester, UK.

Ankolekar, A., Krötzsch, M., Tran, T., \& Vrandecic, D. (2007, May). The two cultures: Mashing up Web 2.0 and the Semantic Web. In Proceedings of the 16th international conference on World Wide Web, 825-834.

Assar, S., Amrani, R.E., \&Watson, R.T. (2010). ICT and Education: A Critical Role in Human and Social Development. Information Technology for Development, 151-158.

Bider, I., Johannesson, P., \& Perjons, E. (2013). Design Science Research as Movement Between Individual and Generic Situation-Problem-Solution Spaces. Designing Organizational Systems, 35-61. 
Collis, B., \& Wende M. (2002). Models of Technology and Change in Higher Education: an International Comparative Survey on the Current and Future Use of ICT in Higher Education (Report). Retrieved from: http://doc.utwente.nl/44770/1/Collis02models.pdf

Democratichni Iniciativi and International Renaissance Foundation. (2011). Corruption in Higher Education Institutions (sociological study report). Retrieved from http://dif.org.ua/modules/pages/files/1345098675_1911.pdf.

Dill, D.D., \& Soo, M. (2004). Transparency and Quality in Higher Education Markets, Markets in Higher Education Rhetoric or Reality, Springer Netherlands, 61-86.

Eisler, D.L. (2001) Campus Portals: Supportive Mechanisms for University Communication, Collaboration, and Organizational Change. Journal of Computing in Higher Education, 13 (1), 3-24.

European Association for Quality Assurance in Higher Education. (2009). ENQA report on Standards and Guidelines for Quality Assurance in the European Higher Education Area. Retrieved from http://www.enqa.eu/files/ESG_3edition (2).pdf

European University Association. (2010). EUA Policy Statement on Quality and Quality Assurance in the European Higher Education Area [Policy Position Document]. Retrieved from http://www.eua.be/Libraries/Publications_homepage_list/EUA-QAPolicy-2010.sflb.ashx.

Garcia-Murillo M. (2013). Does a government web presence reduce perceptions of corruption?, Information Technology for Development, 151-175.

Gryshko, S. (2012) Trust as main paradigm of QA in HE of Ukraine. Higher education of Ukraine, 3(2), 49-52.

Hanushek, E., \& Wößmann, L. (2007). The Role of Education Quality for Economic Growth, World Bank Policy Research Working Paper, 4122. Retrieved from: http://openknowledge.worldbank.org/bitstream/handle/10986/7154/wps4122.pdf?sequ ence $=1$.

Harvey, L., \& Williams, J. (2010). Fifteen years of quality in higher education. Quality in Higher Education, 16, 3-36.

Harvey, L. (2011) Twenty years of trying to make sense of quality assurance; the misalignment of quality assurance with institutional quality frameworks and quality culture. Retrieved from: http://www.eua.be/Libraries/EQAF_2010. WGSII_7_Papers _Harvey. sflb. ashx

Hevner, A.R., March, S.T., \& Park, J. (2004) Design Science in Information Systems Research. MIS Quarterly, 28(1), 75-105.

Hoecht, A. (2006). Quality assurance in UK higher education: Issues of trust, control, professional autonomy and accountability. Higher Education, 51(4), 541-563.

Jafari, A. \& Sheehan, M. (2003) Designing Portals: Opportunities and Challenges. Information Science Pub.

Katz, R.N. (2002). Web Portals and Higher Education. San-Francisco, CA: Jossey-Bass.

Klyne, G., Carroll, J. J., \& McBride, B. (2004). Resource description framework (RDF): Concepts and abstract syntax. W3C recommendation, 10.

Kvit, S. (2012, July 8). Country would benefit from Further European Integration. University World News. Retrieved from http://www.universityworldnews.com/article.php?story=20120705122144461.

Lee, S., Lee, M., Kim, P., Jung, H., \& Sung, W. K. (2010). OntoFrame s3: semantic webbased academic research information portal service empowered by STAR-WIN. In The Semantic Web: Research and Applications (pp. 401-405). Springer Berlin Heidelberg. 
Leveille, D.E. (2006). Accountability in Higher Education: a Public Agenda for Trust and Cultural Change (Visiting Scholar Report). Retrieved from http://cshe.berkeley.edu/publications/docs/Leveille_Accountability.20.06.pdf.

Maedche, A., Staab, S., Stojanovic, N., Studer, R., \& Sure, Y. (2003). Semantic portal-the seal approach. Spinning the Semantic Web, 317-359.

Mankiw, N.G., Romer, D., \& Weil, D. (1992). A Contribution to the Empirics of Economic Growth. Quarterly Journal of Economics, 107(2), 407-37.

McGuinness, D. L., \& Van Harmelen, F. (2004). OWL web ontology language overview. W3C recommendation, 10(2004-03), 10.

Mühleisen, H., Walther, T., \& Tolksdorf, R. (2011). A survey on self-organized semantic storage. International Journal of Web Information Systems, 7(3), 205-222.

Negash, S. (2010). Learning assessment of a videoconference-based training: lessons from medical training between USA and Ethiopia. Information Technology for Development, 212-231.

Nkansah, G.B., \& Unwin, T. (2010). The contribution of ICTs to the delivery of special educational needs in Ghana: practices and potential. Information Technology for Development, 191-211.

Osipian, A. (2008). Political graft and education corruption in Ukraine: compliance, collusion, and control. Demokratizatsiya: the journal of post-Soviet democratization, 16(4), 323 344.

Osipian, A. L. (2009). Corruption and reform in higher education in Ukraine. Canadian and International Education/Education canadienne et internationale, 38(2), 104-122.

Parliament Committee on Education and Science of Ukraine. (2013). Draft Regulation on the Commission of the Future of the Verkhovna Rada of Ukraine [Draft regulation]. Retrieved from http://w1.c1.rada.gov.ua/pls/zweb2/webproc4_1?pf3511=47180

Parusinski, J. (2010). Ukraine's Higher Education Institutions: Fighting isolation. European Focus. Retrieved from http://www.icps.com.ua/files/articles/56/10/EF_7_2010_ENG.pdf

Reynolds, D., Shabajee, P., \& Cayzer, S. (2004, May). Semantic information portals. In Proceedings of the 13th international World Wide Web conference on Alternate track papers \& posters (pp. 290-291). ACM.

Schleicher, A. (2006). The Economics of Knowledge: Why Education is Key for Europe's Success. Lisbon, Portugal: Lisbon Council. Retrieved from http://www.oecd.org/dataoecd/43/11/36278531.pdf.

Skopik, F., Truong, H. L., \& Dustdar, S. (2009). Trust and reputation mining in professional virtual communities. In Web Engineering (pp. 76-90). Springer Berlin Heidelberg.

Shraim, K., \& Khlaif, Z. (2010). An e-learning approach to secondary education in Palestine: opportunities and challenges. Information Technology for Development, 159-173.

State Educational Inspection Unit. (2013). SEIU of Ukraine is approbating new approximate criteria set for HEI performance assessment. Retrieved from http://dinz.gov.ua/index/ua/material/351

Stensaker, B. R. (2003). Trance, transparency and transformation: the impact of external quality monitoring on higher education. Quality in higher education, 9(2), 151-159.

Streeten, Paul (May 1994). Human Development: Means and Ends. Human Development (84.2): 232-237.

Temple, P., \& Billing, D. (2003). Higher education quality assurance organisations in Central and Eastern Europe. Quality in Higher Education, 9(3), 243-258.

Terziyan, V. (2011). TRUST: Towards Trust in Quality Assurance Systems. Brief Introduction of the Project Idea [Presentation during the: TRUST (516935-TEMPUS- 
1-2011) Project Coordination Meeting]. Retrieved from http://www.cs.jyu.fi/ai/Quality-2.ppt .

Terziyan, V., \& Kaykova, O. (2012). From Linked Data and Business Intelligence to Executable Reality. International Journal on Advances in Intelligent Systems, 194208.

Tiropanis, T., Davis, H., Millard, D, \& Weal, M. (2009). Semantic Technologies for Learning and Teaching in the Web 2.0 Era. IEEE Intelligent Systems, 24 (6), 49-53.

Tomusk, V. (2000). When East meets West: decontextualizing the quality of East European higher education. Quality in higher education, 6(3), 175-185.

Towards Trust in Quality Assurance Systems. (2012). The QA Concept for HE of Ukraine [Project deliverable]. Retrieved from http://dovira.eu/trac/attachment/wiki/wp1/QA_Concept_for_HE_of_Ukraine.pdf

UNESCO. (2011). Everyone has the Right to Education [UNESCO Education Brochure]. Retrieved from http://unesdoc.unesco.org/images/0021/002127/212715e.pdf.

UNITER/USAID. (2011). The Status of Corruption in Ukraine: Comparative Analysis of National Studies: 2007-2009 (Sociological Study Report). Retrieved from http://uniter.org.ua/data/block/corruption_in_ukraine_2007-2009_2011_ukr.pdf.

Van der Wende, M. C. (2000). The Bologna Declaration: Enhancing the transparency and competitiveness of European higher education. Higher education in Europe, 25(3), 305-310.

Weisberg, H.F. (1974). Models of Statistical Relationship. American Political Science Review, 1638-1655.

Welzel, C., Inglehart, R., \& Kligemann, H.-D. (2003). The Theory of Human Development: A Cross-Cultural Analysis. European Journal of Political Research, 42 (1), 341-379. 